\title{
DIAGNOSTICS OF LIVER HYDATID DISEASE
}

\author{
Valentin Ignatov, Shteryo Shterev, Anton Tonev, Nikola Kolev, Elitsa Encheva. \\ Aleksandar Zlatarov, Krasimir Ivanov
}

Department of surgery, Medical university of Varna

\begin{abstract}
E. granulosis is the species that prevails in most countries around the world and especially in the Mediterranean region. It is one of the most severe infestations in humans. The biological diagnosis is based on serological tests. Eosinophilia is considered variable because it does occur in cases of a hydatid cyst disease but its absence does not exclude the possibility of a hydatid cyst existence. Imaging diagnostics are important for the classification of the hydatid cysts. Ultrasound is a noninvasive, widely available method with high sensitivity to the diagnosis of hepatic echinococcosis and of low price, therefore it is a method of choice. CT is the method of doubt for a suppuration of a hydatid cyst. An MRI with high contrast resolution of T1 and T2 images demonstrated better pericyst, daughter cysts and matrix. We have reviewed the current trends in the diagnosis of liver cystic echinococcosis.
\end{abstract}

Keywords: hydatid cyst, liver echinococcosis, ELISA reaction, hepatic echinococcosis, diagnostic, E. granulosis

\section{INTRODUCTION}

The hydatid cyst disease is a cosmopolitan parasitosis (1). E. granulosis is the species that prevails in most countries around the world and especially in the Mediterranean region $(1,2)$. It is one of the most severe infestations in humans. Compared to other zoonosis, the hydatic cyst disease, caused by E.granulosus, most often leads to prolonged disablement, frequent recurrences and is associated with permanent disability and high mortality rates, which makes it a serious medico-social and economic problem for our country. Data from the literature suggest that the liver is the most frequently affected organ.

\footnotetext{
Address for correspondence:

Georgi Ivanov

First Clinic of Surgery, University Hospital "St. Marina"

$1 \mathrm{Hr}$. Smirnenski Str.

9000, Varna, Bulgaria

Medical University of Varna

E-mail:ghivanov@abv.bg
}

Received: September 30, 2013

Accepted: December 18, 2013
(3). The standard diagnostic approach for the hydatid cyst disease of the liver includes a combination of imaging techniques and laboratory and serological analysis $(3,4)$.

\section{LABORATORY METHODS}

Diagnosis can be confirmed by a series of biological tests that are based on the reaction of the host against the parasite. Eosinophilia is considered variable because it does occur in cases of a hydatid cyst disease but its absence does not exclude the possibility of a hydatid cyst existence. It is considered that a significant eosinophilia occurs in over $50 \%$ of all cases $(1,2,3,21)$. Routine laboratory findings are not specific and therefore can show normal or abnormal results $(3,5,6,22)$. ELISA reaction, immunofluorescent reaction, passive haemagglutination reaction, latex agglutination reaction, immunoelectroforesis reaction, double diffusion in gel reaction. Complement adhesion reaction has a bad sensitivity and specificity. Indirect haemagglutinaton reaction is shown to have good sensitivity but there is also a big percentage of false positive results. Immunoelectroforesis and ELISA according to different authors show 
the best results with sensitivity up to $90 \%$ for hydatid cysts located in the liver (3-6).

The immunologic diagnostic techniques include primary screening and confirmation tests. Non-sensitive and non-specific tests such as the intradermal test of Cassoni, complement adhesion test, indirect haemagglutination test and latex- agglutination test have been replaced by ELISA, indirect immunofluorescent test for antibodies (IFAT), immunoelectroforesis (IEP) and immunoblot (IB) in the routine laboratory practice $(5,6)$. Screening tests with ELISA use crude antigens and are associated with a high incidence of false negative and false positive results. However, findings of specific antigens and immune complexes with ELISA according to different authors have a $95 \%$ sensitivity, but the specificity is relatively low - $61.7 \%(6,13,20,21)$.

Biological diagnosis is based on serological tests.

Sbihi et al. reported that purification procedures can have a strong influence on the diagnostic value of antigens with identical electrophoretic behaviour. Purified fractions enriched in antigens 5 and $\mathrm{B}$ and glycoproteins from hydatid fluid increased sensitivity to $95 \%$ and specificity - to $100 \%$ $(7,9)$. According to other authors in studies with the use of purified fractions - ten to twenty percent of the patients with liver cysts do not form echinoccocus-specific serum antibodies (IgG) and thus give false negative results $(8,9,22)$. There is an association between calcifying massive hydatid cysts of the liver and negative serological tests. In a study with the help of indirect haemagglutination it was found that false negative results occur in patients with cysts of sizes smaller than $9 \mathrm{~cm}$, intact cysts, cysts with extrahepatic locations and unilocular single cysts $(7,17,19)$.

Furthermore, $10 \%$ to $15 \%$ of false positive results are obtained because of cross-reactions with other helminth infections (cysticercosis), as well as non-infectious diseases (cancer, autoimmune diseases, pregnancy) (7). Immunological diagnostic methods showed positive results in $75 \%-90 \%$ of patients, therefore proving insufficient as a single test for the exact diagnosis. To lower the false-negative and falsepositive results a combined diagnostic approach (imaging and lab) could be applied $(8,9,15,17,19)$.

\section{IMAGING METHODS Ultrasonography}

Ultrasound is a noninvasive, widely available method with high sensitivity to the diagnosis of hepatic echinococcosis and of low price, therefore it is a method of choice $(16,2)$. Many authors have suggested ultrasound models and classification of hepatic echinococcosis. Ultrasound is useful for determining the internal structure, size, number and location of cysts, the presence of complications with a sensitivity of $90 \%-98 \%$ according to the available literature $(10,20)$.

The method is useful for planning appropriate treatment and evaluation of the therapy. Several comparative studies have shown that ultrasound is more accurate in the detection of cysts than immunodiagnostic tests since the latter show relatively high rate of false negative and false positive results. (10). In one study, in $97 \%$ of the patients ultrasound allows not only to diagnose, but also to perform accurate assessment of possible variations of the biliary tract and any present complications $(10,12,15,20)$.

\section{Computed tomography}

Computed tomography (CT) provides information equivalent to that of a conventional or intraoperative ultrasonography but shows a more detailed location and depth of the available hydatid cyst. With CT one can also determine the presence of exogenous daughter cysts and to determine the consistency of the cyst. This diagnostic method is essential in planning appropriate surgical treatment especially in laparoscopic surgery. CT is used in cases in which ultrasound is difficult, e.g. due to obesity, intestinal gas, a deformed abdominal wall, previous surgery or medical complications. CT is highly sensitive and a specific method of exploration of a hepatic hydatid cyst (11). Intravenous contrast is not routinely used unless there is suspicion of probable suppuration in the communication with the biliary tree. (10) Improved resolution with contrast enhancement is important in the diagnosis of the hepatic hydatid cyst density between the cyst and the normal liver parenchyma. Cystic fluid usually shows hypoechogenicity (3-30 HU) (12). CT easily detects a calcification of the cystic wall or the internal septa. In CT without contrast the hydatid cyst is visualised with a 
hyperechogenic wall, even without calcification (20). There is a better visualisation of the differentiation of the cuticular membrane of the fibrous capsule perycyst $(11,12)$. Daughter cysts appear as round structures located peripherally within the mother cyst. They usually contain liquid with lower echogenicity than the cyst fluid in the maternal one (10). According to Mortele $\mathrm{KJ}$ and associates, large calcifications are found in $50 \%$ of the cases, and daughter cysts occur in approximately $75 \%$ (11). CT is the method of doubt for suppuration of the hydatid cyst $(11,12,20)$.

According to Agayev CT has 100\% accuracy in the diagnosis of liver hydatid disease (11). According to Suwan Z. CT was superior to ultrasound in the visualisation of gas cysts, calcifications, and more precisely in determining the position of hydatid cysts. (10). Numerous studies confirm the higher sensitivity of CT compared to that of ultrasound in the diagnosis of hydatid disease (12).

According to various authors in the literature, false-negative results, when administering CT, are observed at a higher rate in patients with fatty degeneration of the liver parenchyma. Having similar density, echinoccocus cyst and fat, even hamper the research and administration of intravenous contrast. $\mathrm{CT}$ is a more reliable method to detect small cysts when compared to ultrasound (10).

\section{MRI}

MRI gives better structural details of the echinococcus cyst, however its use is not economically viable. According to various authors there is no significant superiority of the method in the diagnosis of hepatic echinococcosis and therefore it is not applied everywhere. The administration of gadolinium gives a high rate of detection of echinoccocus cysts demonstrated in T1 and T2 images (12). MRI with high contrast resolution of T1 and T2 images demonstrated better pericyst, daughter cysts and matrix (13). On T1 and T2 images a pericyst is seen as the same ring due to its fibrous structure and the presence of calcifications. Hydatid matrix is presented as the same structure on T1 images and significantly the same structure on T2 images. Daughter cysts were reported as structures similar to the matrix of the breast cyst on $\mathrm{T} 1$ and $\mathrm{T} 2$ images $(12,14,18)$.
Sagittal and coronary slices of magnetic resonance imaging demonstrated a well-invaded echinoccocus cyst through the diaphragm. The main advantage of MRI over CT and ultrasound is in severe cases of complicated hydatid disease with a suspected rupture of the cyst into the biliary tree (14). MRI findings of a rupture of the echinococcus cyst are divided into direct (a break in the cuticle and a leakage of cyst fluid) and indirect (increased echogenicity, presence of gas in the cyst, a change in the intensity image) $(12,13,14,23)$.

\section{CONCLUSION}

Cystic echinococcosis is a zoonotic disease caused by an infection with Echinococcus granulosus. The immunological diagnostic methods show variable results, therefore proving insufficient as a single test for the exact diagnosis. To reduce the falsenegative and false-positive results, a standard diagnostic approach to hepatic hydatid disease involves a combination of imaging techniques and laboratory and serological analysis.

\section{REFERENCES}

1. Mandal S, Mandal MD. Human cystic echinococcosis: epidemiologic, zoonotic, clinical, diagnostic and therapeutic aspects. Asian Pac J Trop Med. 2012 Apr;5(4):253-60.

2. Reid-Lombardo KM, Khan S, Sclabas G. Hepatic cysts and liver abscess. Surg Clin North Am. 2010 Aug;90(4):679-97.

3. Plard L, Guedin P, Le Pennec V, Chiche L. Hepatic cysts: diagnosis and management. J Chir (Paris). 2008 May-Jun;145(3):217-25.

4. Cubas-Castillo R, Brain CO, López-Guillemain R, Ballarino EA Hydatid liver disease: our 25-year experience. Cir Cir. 2011 Jul-Aug;79(4):306-312. English, Spanish

5. Agaba EA, Camacho DR, Vemulapalli P.Hydatid cyst disease revisited: optimal management of complex liver disease. South Med J. 2011 Apr;104(4):254-5. No abstract available.

6. Parlakgumus A, Ezer A, Calhshan K, Colakoglu T, Belli S, Karakaya J, Yildirim S.Effects of a preoperative and perioperative multidisciplinary approach for complicated liver hydatidcyst on postoperative outcomes. Hepatogastroenterology. 2010 Nov-Dec;57(104):1493-8. 
7. Sbihi Y, Janssen D, Osuna A. Specific recognition of hydatid cyst antigens by serum IgG, IgE, and IgA using western blot. J Clin Lab Anal. 1997;11(3):154-7.

8. Sbihi Y, Rmiqui A, Rodriguez-Cabezas MN, Orduña A, Rodriguez-Torres A, Osuna A.

Comparative sensitivity of six serological tests and diagnostic value of ELISA using purified antigen in hydatidosis. J Clin Lab Anal. 2001;15(1):14-8.

9. Yalcin E, Kiper N, Tan C, Ozcelik U, Dogru D, Cobanoglu N, Kose M, Pekcan S, Aslan AT, Ersoy F.The role of human leucocyte antigens in children with hydatid disease: their association with clinical condition and prognosis. Parasitol Res. 2010 Mar;106(4):795-800. Epub 2010 Jan 29

10. Moore J, Gupta V, Ahmed MY, Gociman B. Hydatid cyst disease: optimal management of complex liver involvement. South Med J. 2011 Mar;104(3):222-4

11. Wen H, Aji T, Shao YM. Diagnosis and management against the complications of human cystic echinococcosis. Front Med China. 2010 Dec;4(4):394-8. Epub 2010 Dec 1

12. Oruç E, Yıldırım N, Topal NB, Kılıçturgay S, Akgöz S, Savcı G.The role of diffusion-weighted MRI in the classification of liver hydatid cysts and differentiation of simple cysts and abscesses from hydatid cysts. Diagn Interv Radiol. 2010 Dec;16(4):279-87. Epub 2010 Jun 26.

13. Heirwegh G, Claikens B.Type I hydatid cyst of the liver: typical MRI features. JBR-BTR. 2005 May-Jun;88(3):136-7

14. Kumar R, Reddy SN, Thulkar S. Intrabiliary rupture of hydatid cyst: diagnosis with MRI and hepatobiliary isotope study. Br J Radiol. 2002 Mar;75(891):271-4. 